\title{
Single-grade engine lubricant: introduce viscosity index numerical order (VIno)
}

\begin{abstract}
Single-grade engine oil viscosity experience changes influenced by temperature and pressure. However, in reality, the viscosity of engine oil is also affected by age and it has been proven in earlier studies through the viscosity model of the UKM Equation (dUKM). In this mathematical model, the viscosity of the engine oil is manipulated by the factors of temperatue and time. Both of this factors relies fully on laboratory data. Based on this hypothesis, a model of the viscosity which is based on a mathematical model have been developed whereby the technical temperature factor value and technical time factor are obtained through engine oils technical data such as the density, viscosity index and kinematic viscosity; technical parameter displayed in technical statements of every engine oil. This model is named the viscosity model of viscosity index numerical order ( $\mathrm{dVIno}$ ) and the estimation of viscosity obtained through the dVIno was found to not exhibit any significant deviation from the actual viscosity of the oil.
\end{abstract}

Keyword: Single-grade engine oil; Technical temperature factor; Technical time factor; UKM Eq. viscosity; VI numerical order viscosity 